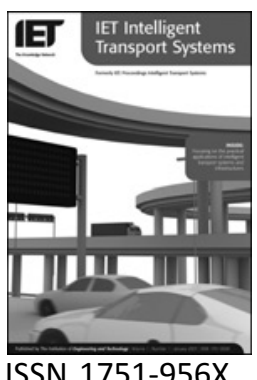

Special Issue - selected papers from HCD 2008

\title{
Drivers' reactions to sudden braking by lead car under varying workload conditions; towards a driver support system
}

\author{
$\begin{array}{lll}\text { T.W. Schaap } & \text { A.R.A. van der Horst } & \text { B. van Arem } \\ & 1,3\end{array}$ \\ K.A. Brookhuis ${ }^{4,5}$
}

${ }^{1}$ Centre for Transport Studies, Research Centre AIDA (Applications of Integrated Driver Assistance), University of Twente, PO Box 217, 7500 AE Enschede, The Netherlands

${ }^{2}$ TNO Defence, Security and Safety, PO Box 23, 3769 ZG Soesterberg, The Netherlands

${ }^{3}$ TNO Built Environment and Geosciences, PO Box 49, 2600 AA Delft, The Netherlands

${ }^{4}$ Faculty Technology, Policy and Management, Delft University of Technology, PO Box 5015, 2600 GA Delft, The Netherlands ${ }^{5}$ Department of Psychology, University of Groningen, Grote Kruisstraat 2-1, 9712 TS Groningen, The Netherlands

E-mail: t.w.schaap@utwente.nl

\begin{abstract}
At urban intersections drivers handle multiple tasks simultaneously, making urban driving a complex task. An advanced driver assistance system may support drivers in this specific driving task, but the design details of such a system need to be determined before they can be fully deployed. A driving simulator experiment was conducted to determine the relationship between different subtasks of driving at urban intersections. Participants completed four drives, each comprising 20 comparable intersections with different traffic situations and encountered one unexpected braking event during the experiment. The effects of varying levels of event urgency on the relationship between different driving subtasks were studied. Furthermore, the influence of workload on this relationship was determined by giving half of the subjects an additional cognitive task. After the lead car braked unexpectedly, participants reduced speed and increased headway depending on the urgency of the braking event. Depending on the workload, participants returned to the normal speed and headway again after a number of intersections. Participants experiencing a high-workload drove more smoothly, except for those who had experienced the most urgent unexpected event. High workload additionally affected the length of the adjustments to the unexpected event.
\end{abstract}

\section{Introduction}

Driving safely requires effort and a wide range of skills. Urban intersections establish demanding situations for drivers in this sense, as can be concluded from crash data; $38.5 \%$ of all Dutch traffic accidents leading to injuries in 2007 occurred at urban intersections [1]. A partial explanation for this can be found in the complexity of the driving task at intersections. Urban driving involves not only the correct use of vehicle controls, but also planning tasks and decisions on specific manoeuvres. For instance, drivers have to take turns, avoid collisions with vehicles crossing their lane at intersections and keep an appropriate distance from other roads users. In addition to all these subtasks that together constitute the primary task of driving, drivers nowadays are confronted with many secondary tasks that demand attention, such as talking on a mobile phone or handling a navigation system. These tasks may interfere with the primary driving task $[2,3]$.

The complexity of the driving task becomes clear when looking at novice drivers; all subtasks are new and require full attention, although juggling all these tasks simultaneously seems almost impossible to do. With driving experience, skills are developed until a large part of the multiple driving tasks can be performed in an almost automatic way. 
Nevertheless, many subtasks still require attention, goal management and the use of working memory.

Advanced driver assistance systems (ADAS) provide promising ways towards a safer and more efficient traffic system, with more comfort for the driver [4-6]. However, drivers' needs are not always the focus of the designing process of ADAS; many ADAS developments are technology driven. Adding a new system to the humanvehicle interaction changes the driving task, and may therefore change the way drivers perceive and perform their task. This behavioural adaptation may in certain cases bring about unwanted changes, such as adaptation of safety margins and speed ([7] provides an overview), and an increase in reaction time $[8,9]$. A well-guided process of development and evaluation of ADAS is therefore an important step towards safer driving.

This paper presents the results of a driving simulator study conducted to investigate how different subtasks within the driving task are related to each other at intersections, and how different factors influence the relationship between these different subtasks. Following a review of what is already known about the structure of the driving task, the aim, set-up and results of the driving simulator study are presented in consecutive sections.

\section{Background: driving task}

Almost all subtasks within the driving task affect other tasks through the outcome of their actions. Some tasks, such as deciding when and where to drive, might take place over a long time period, whereas others, such as sudden braking or a glance in the rear-view mirror, often take less than a second. This relationship between different types of subtasks has been classified by Michon [10], describing a hierarchical three-layer model of the driving task, in which the driving task is divided into three levels: strategic, tactical and operational. At the strategic level, the goals of the trip, as well as destination, route, general speed and thus departure and arrival time, are decided upon. At the tactical level, the driver interacts with other road users and the environment. Among the things this entails are maintaining a steady speed, keeping a safe distance from other vehicles and staying within the correct lane. At the operational level, the driver controls the vehicle by pressing the pedals, turning the steering wheel, changing gear (in a manual clutch system) - in other words, using the vehicle controls. At urban intersections, for instance, route choice, tactical manoeuvres and control tasks are all important at the same time, whereas motives concerning the trip (purpose, duration, importance) or situations encountered during the trip (unsafe or unexpected situations) may influence behaviour on other levels. When a certain route has been chosen, drivers have to make the manoeuvres according to the route and control the vehicle to follow the chosen road. This top-down influence (or anticipation [10]) occurs during normal driving. Bottom-up influence,

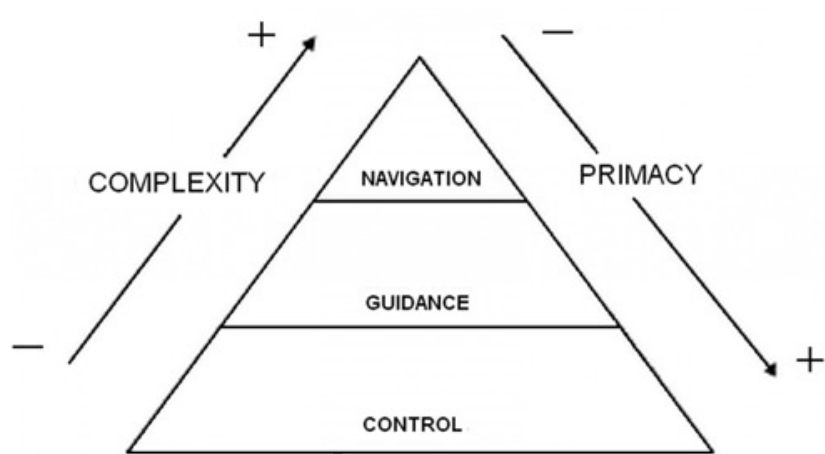

Figure 1 Complexity and primacy at different levels of the drinking task, after [12]

on the other hand, is prompted by unexpected situations. Situations encountered during the trip may create or influence shorter-term goals when lower-level tasks have to guarantee safety by compensating or when the original task cannot be performed successfully [11]. In these cases, lower-level actions influence the goals of higher-level tasks.

To understand how this interaction works, it is useful to be aware of how the various subtasks in driving are related and in which ways they are different from each other. Alexander and Lunenfeld [12] describe how the layers of the driving task differ in both complexity and primacy. They state that primacy (i.e. is the priority of dealing with a certain task at a certain moment) increases with lower-level tasks, whereas complexity increases with higher-level tasks (Fig. 1).

Tasks on a high level often take more time to complete and are more complex, but do not have a direct impact on safety. On the other hand, correct execution of lower-level tasks is crucial for safe driving, but these tasks can mostly be performed in a more or less automatic way. Higher-level tasks such as the goal of the trip or the interaction with the road layout receive most of the directed attention in a regular driving situation. Nevertheless, in unexpected situations such as a slippery road, lower-level tasks have to be activated and take priority over higher-level tasks to ensure safety. The latter situations are of particular interest for the present research because drivers then compensate for unexpected events, constituting the bottom-up influence between levels of the driving task. This is an influential but largely unexplored process in the structure of the driving task.

\section{Aim and research questions}

The aim of the research project is to determine which type of driver support drivers need in order to secure their safety, and to study behavioural adaptation to a driver support system. To this end, it is necessary to understand the structure of the driving task and the ways in which different subtasks are handled.

The following research questions concern the interaction between the levels of the driving task. 
- When and how does bottom-up influence occur among the different driving task levels?

- To what extent does additional cognitive workload affect the interaction between subtasks of driving?

- To what extent does the urgency of an unexpected event change this interaction?

An unexpected event is expected to cause compensation behaviour on a lower-level task, temporarily influencing higher-level tasks. After a while, the effect of the unexpected event will dissipate. Additional cognitive workload might interfere with the driver's ability to fully comprehend the current situation and affect decisions about actions to be taken [13], although the urgency of the event might have an influence on the level of compensation behaviour needed. Therefore the study includes high workload from a secondary task and varying level of event urgency that may influence the adjustments in tactical behaviour after an unexpected event.

\section{Method}

\subsection{Participants}

The analysis is based on complete data sets of 39 subjects who participated in the experiment. They were between 23 and 60 years old, had their driver's licence for at least 5 years and drove $7000 \mathrm{~km}$ or more annually. The average age of the 39 participants was 41 years (SD 13, minimum 23, maximum 60); 26 participants were male and 13 female. All participants were paid $€ 18$ for their participation. Most of the participants had prior experience in driving simulator studies.

\subsection{Data measurement}

The experiment was performed in a TNO human factors fixed-based driving simulator with manual transmission (Fig. 2a). The participant was able to control the driving simulator by means of normal vehicle controls. The road environment and other road users were projected onto

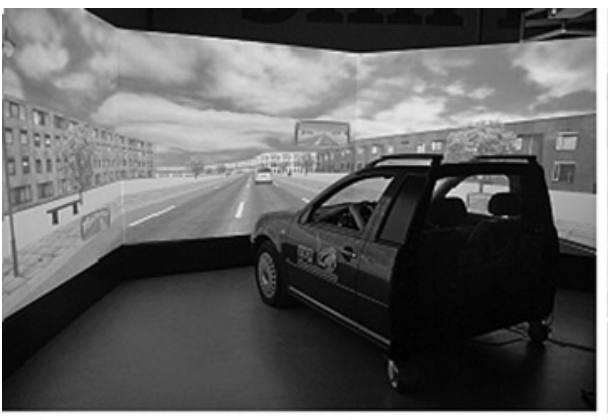

$a$

Figure 2 TNO fixed-based driving simulator

$a$ General setup

$b$ Layout and positioning of other road users at each intersection three screens with a total horizontal field of view of $180^{\circ}$ and total vertical field of view of $45^{\circ}$ [14]. The experimental environment and scenarios were developed using specific graphics software by ST Software [15]. The traffic participants in the virtual environment (Fig. 2b) are autonomous agents that by default interact with all other participants (including the experimental vehicle) and the road environment according to Dutch traffic rules [15].

To assess the participants' workload during the experiment, the participants were required to perform the peripheral detection task (PDT) [16] during driving. The PDT is based on the premise that visual attention narrows as the workload increases [16]. Participants wear a headband with an LED light, which lights up randomly every 3-5 s. Participants are instructed to press a switch attached to their index finger as soon as they see the LED signal. As workload increases, response time and the chance of missing a signal also increases [16]. The workload is monitored by response times and the number of missed signals (defined as a response time of $2 \mathrm{~s}$ or more). Fig. 3 shows the headband with the LED (Fig. $3 a$ ) and the switch on the index finger (Fig. 3b).

The PDT has disadvantages and advantages over other measures of workload, such as self-report measures or physiological measures. The main disadvantage of self-report measures such as the NASA-TLX [17] is that they are subjective [18], whereas only an overall rating of workload over a completed ride can be given. Physiological measurements are objective and unobtrusive [19], but require complex interpretation of results and may be contaminated, for instance, by emotional strain and physical activity [20]. The PDT has been shown to be a reliable indicator of the demands of the driving task [16], is easy to interpret and is sensitive to peaks in workload [20], nevertheless, it is a secondary task requiring some attention. The repeatability and ability of the PDT, especially the missed signal rate, to distinguish between levels of workload was found in [21]. Finally, the PDT was also established as a tool for visual distraction and mental workload in the real road-traffic environment [22].

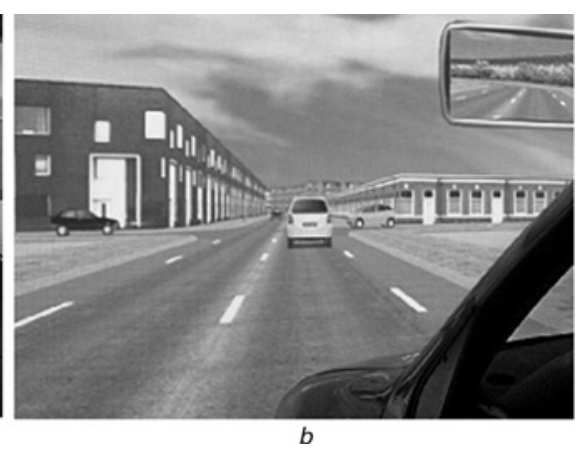




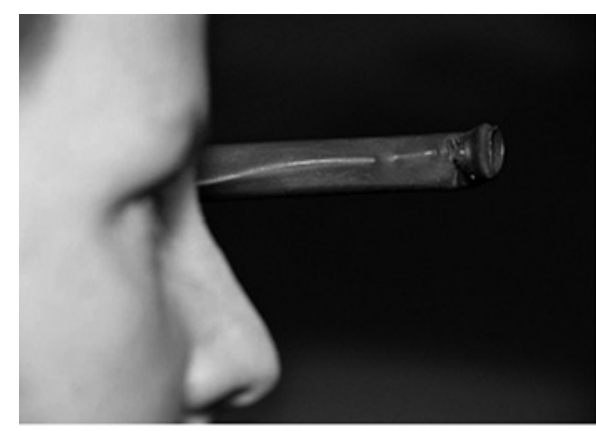

a

Figure 3 Peripheral Detection Task (PDT)

a Headband with LED

$b$ Switch on index finger

\subsection{Experimental design}

An urban layout was simulated as one long road with 20 fourway intersections. Subjects were instructed to drive straight on each intersection with a maximum speed of $50 \mathrm{~km} / \mathrm{h}$ and give priority to traffic coming from the right according to Dutch traffic regulations. Each of the 21 road sections was at least $250 \mathrm{~m}$ long, and one complete drive took an average of $91 / 2 \mathrm{~min}$. The infrastructure at each intersection was comparable. A lead car drove in front of the subject during the whole drive, slowing down when the subject fell too far behind (more than $48 \mathrm{~m}$ away) and speeding up when the subject came too close (within $18 \mathrm{~m}$ ). When the participant was approaching an intersection, a car coming from the right always crossed the intersection first. The participant and a lead car then reached the intersection, and a car from the left approached the intersection, yielded to the participant and the lead car, and crossed behind them. These actions of the other road users were similar at each intersection. The intersection layout and the positioning of other road users when the participant was about to cross an intersection are depicted in Fig. $2 b$.

The experiment consisted of four such experimental trials, and one introduction drive preceding the actual experiment to give all participants the opportunity to gain some experience with the driving simulator and the simulated environment. In the reference drives, participants drove only on standard intersections, setting their expectations about the situations to come. After five standard intersections in either the second or fourth drive, the lead vehicle braked unexpectedly and then accelerated again to its initial speed after the participant had driven either 5, 10 or $20 \mathrm{~m}$, that is, three levels of urgency, labelled mild, medium and hard. This gave a good indication of the event urgency if participants were at the same distance before the event. However, participants were free (within limits) to choose their own headway at all times (also around the braking event), and the original indicator of urgency did not take into account how close to the lead car the participant was driving. A mild urgency event could therefore potentially be a situation in which the lead car

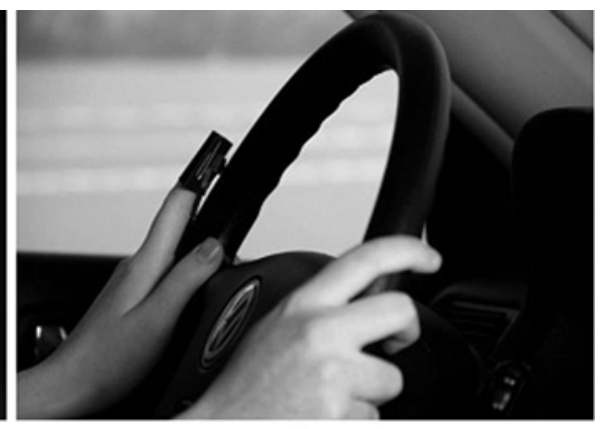

$b$ would brake for only a short time but relatively close to the participant, whereas a high-urgency event could also be a braking lead car far away from the participant. Therefore a second measure of criticality was determined, namely the headway to the lead car after the event had occurred. This measure is more directly related to the possibility of a conflict, and parts of the data were therefore also analysed with this criticality variable. The three criticality levels were set at close (a headway of less than $18 \mathrm{~m}$ ), medium (headway between 18 and $30 \mathrm{~m}$ ) and far (headway over $30 \mathrm{~m}$ ). The order of the trials with the unexpected event and the other (reference) drives was balanced among the participants. The braking event occurred only once for each participant to ensure it was unexpected.

Half of the participants were given an additional cognitive task, to determine the effects of cognitive load on the different levels of the driving task. They were instructed to iteratively subtract a number between 4 and 9 from a threedigit figure between 730 and 850 during each of the four complete drives. Participants were encouraged to continuously perform the additional task and were reminded of their task after a number of seconds without an answer, but they were able to give their final answers at their own pace. Participants were instructed to give the highest priority to the primary driving task. The cognitive task, if applicable, should be given second priority, and the PDT should receive the lowest priority, but should still be completed as fast as possible.

The two levels of cognitive workload (driving only and driving with a secondary task) and the three levels of urgency (mild, medium and hard) combine to six event conditions in a complete between-subject set-up so as to avoid carry-over effects. After each drive, participants were given a break and a questionnaire, with 20 questions about simulator sickness, the predictability of the driving task and whether performing the PDT had interfered with their driving. For the participants with the mathematical task, two additional questions were asked about the level of difficulty of this task, and whether it had influenced the participant's driving style. Participants had to rate their 
answers to the questions on a five-point Likert scale. The questions were phrased as follows:

What did you think of the behaviour of other road users? Please rate your answer on the scale below.

\section{$\begin{array}{lllllll}\text { Predictable } & \mathrm{X} & \mathrm{X} & \mathrm{X} & \mathrm{X} & \mathrm{X} & \text { Unpredictable }\end{array}$}

What did you think of the predictability of the driving task?

$\begin{array}{lllllll}\text { Predictable } & \mathrm{X} & \mathrm{X} & \mathrm{X} & \mathrm{X} & \mathrm{X} & \text { Unpredictable }\end{array}$

Participants had the opportunity to write additional remarks at the end of the questionnaire.

\subsection{Analysis}

Twenty-one dependent variables were registered during the experiment [23], mostly focusing on tactical level tasks such as handling parameters of speed, headway and lateral acceleration. We studied the main effects and two-way interactions by means of a repeated measures analysis of variance (Repeated measures ANOVA) method. The statistical significance level was set at 0.05. Correlations between certain variables were calculated as well.

The following independent variables were studied

- Urgency (3): mild (5 $\mathrm{m}$ driven during braking by the lead car), medium (10 $\mathrm{m}$ driven), hard (20 $\mathrm{m}$ driven)

- Additional workload (2): with or without additional task

- Criticality because of the headway after the braking event (3): close $(<18 \mathrm{~m})$, medium $(18-30 \mathrm{~m})$, far $(>30 \mathrm{~m})$

The dependent variables were compared with respect to

- Drive (2): trial with a braking lead car; reference drive (third drive for all participants)

- Intersection (8): intersections before, during and after the unexpected event

Analyses of the predictability of the drives were based on

- Subject of question (2): predictability of the driving task and predictability of the behaviour of other road users
Table 1 Average speed and standard deviation of speed per drive

\begin{tabular}{|c|c|c|}
\hline Drive & $\begin{array}{c}\text { Average speed, } \\
\mathrm{km} / \mathrm{h}\end{array}$ & $\begin{array}{c}\text { Standard deviation of } \\
\text { speed }\end{array}$ \\
\hline drive 1 & 40.49 & 8.31 \\
\hline drive 2 & 42.48 & 7.09 \\
\hline drive 3 & 44.34 & 8.02 \\
\hline drive 4 & 44.87 & 8.10 \\
\hline
\end{tabular}

- Drive (3): two reference drives (first and third drive for all participants) and trial with the braking lead car.

\section{Results}

\subsection{Expectations and workload}

The expectations of the participants were tested by studying the learning effects in their driving behaviour (i.e. habituation during the four drives). Average driving speed increased during the first drive, whereas the standard deviation of the driving speed decreased after the initial drive (see Table 1 for the size and direction of the speed effects). This supports the expectation that participants got used to the standard intersections during the first experimental drive.

Learning effects were not only seen in a difference in speed and standard deviation of speed after the first drive, but also in the development of speed at the first intersections of each drive. At the first four intersections of each drive, participants increased their average speed from $36.9 \mathrm{~km} / \mathrm{h}$ at the first intersection to $40.6,43.0$ and $43.3 \mathrm{~km} / \mathrm{h}$ at the second, third and fourth intersections, respectively. This was a significant increase $(F(3,99)=33.001, p<0.001)$. Posthoc tests (Tukey's HSD) showed that all intersections differed significantly from each other, except for the third and the fourth. To eliminate the learning effects from the analyses, the first four intersections were not included in the final analyses.

In addition, after the trial with the braking lead car and the first and third drives (both reference drives), participants' answers to two questions concerning the predictability of the driving task and the behaviour of other road users were examined. The first and third drives were selected since these were two reference drives for all participants. The answers to the two different questions did not differ

Table 2 Learning effects and expectations of participants

\begin{tabular}{|l|l|c|c|}
\hline \multicolumn{1}{|c|}{ Variable } & \multicolumn{1}{c|}{ Effect } & $F$-value & $p$-value \\
\hline average speed & four drives & $F(3,99)=12.080$ & $p<0.001$ \\
\hline standard deviation of speed & four drives & $F(3,99)=30.241$ & $p<0.001$ \\
\hline answers to the questionnaire & three drives & $F(2,62)=4.057$ & $p<0.025$ \\
\hline
\end{tabular}




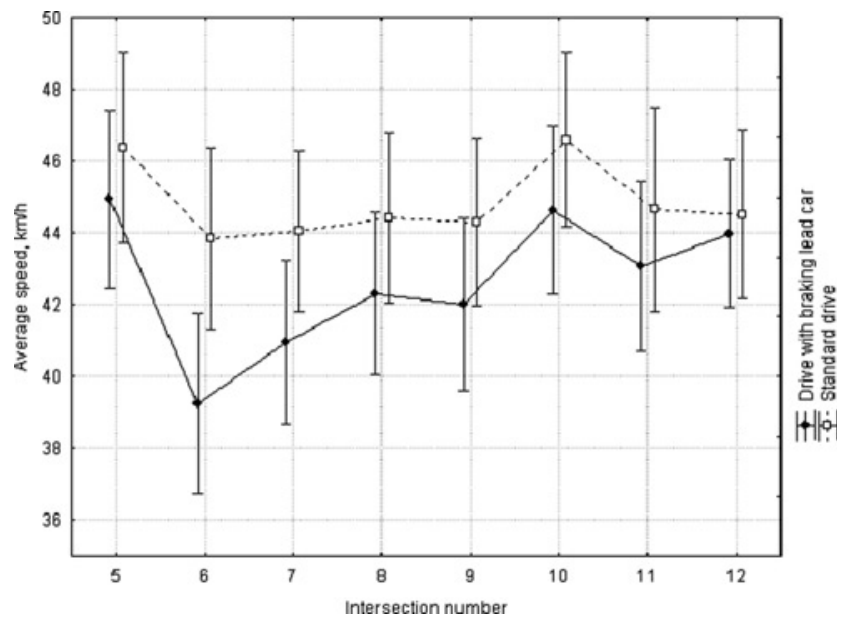

Figure 4 Average speed as a function of drive and intersection

significantly $(F(1,31)<1)$ and the answers were therefore combined in the analyses. The combined answers (two per drive per participant) revealed that the reference conditions were seen as significantly more predictable than the drive with the unexpected braking event. See Table 2 for the learning effects and expectations of the participants.

The effects of the additional cognitive task on workload were established by analysing the data from the PDT. The additional task significantly increased both the reaction times to the PDT $(F(1,29)=54.725, p<0.001)$ and the number of missed signals $(F(1,33)=34.873, p<0.001)$, confirming the hypothesis that the additional task increased the cognitive workload of the drivers. Answers to the questionnaire revealed that the PDT was generally not seen as difficult or interfering with driving; $90 \%$ of the participants responded after each drive that the PDT was easy to perform, and $97 \%$ answered that the PDT had no influence on their driving behaviour.

\subsection{Reactions to the unexpected braking event}

Directly after the braking event (immediately after intersection 5 in drive 2 or 4 ), a number of changes in the participants' tactical driving behaviour occurred. Speed was lowered for the duration of a number of intersections and normalised again in approximately six intersections after the braking event $(F(7,231)=3.235, p<0.005)$. Fig. 4 shows the average speed values per intersection for the reference drive and those for the drive with the braking event. A similar effect was seen in the minimum speed at the intersection area $(F(7,231)=3.304, p<0.005)$. The adjustment in the average speed was different for groups with and without an additional task. Although not much difference was evident between the reference drive and the drive with the braking lead vehicle for participants who drove without an additional task, those who experienced higher workload because of the additional task drove significantly slower in the event drive than in the reference drive $(F(1,33)=4.442, p<0.050)$.

Lateral position was also influenced by the additional task. Outliers with a deviation of over $2.5 \mathrm{~m}$ from the middle of the lane were not included in the analyses. Participants without an additional task drove more to the right side of their lane (average -0.34 , SD 0.09) than drivers with an additional task (average -0.03 , SD 0.10$) \quad(F(1$, 22) $=5.149, p<0.050)$.
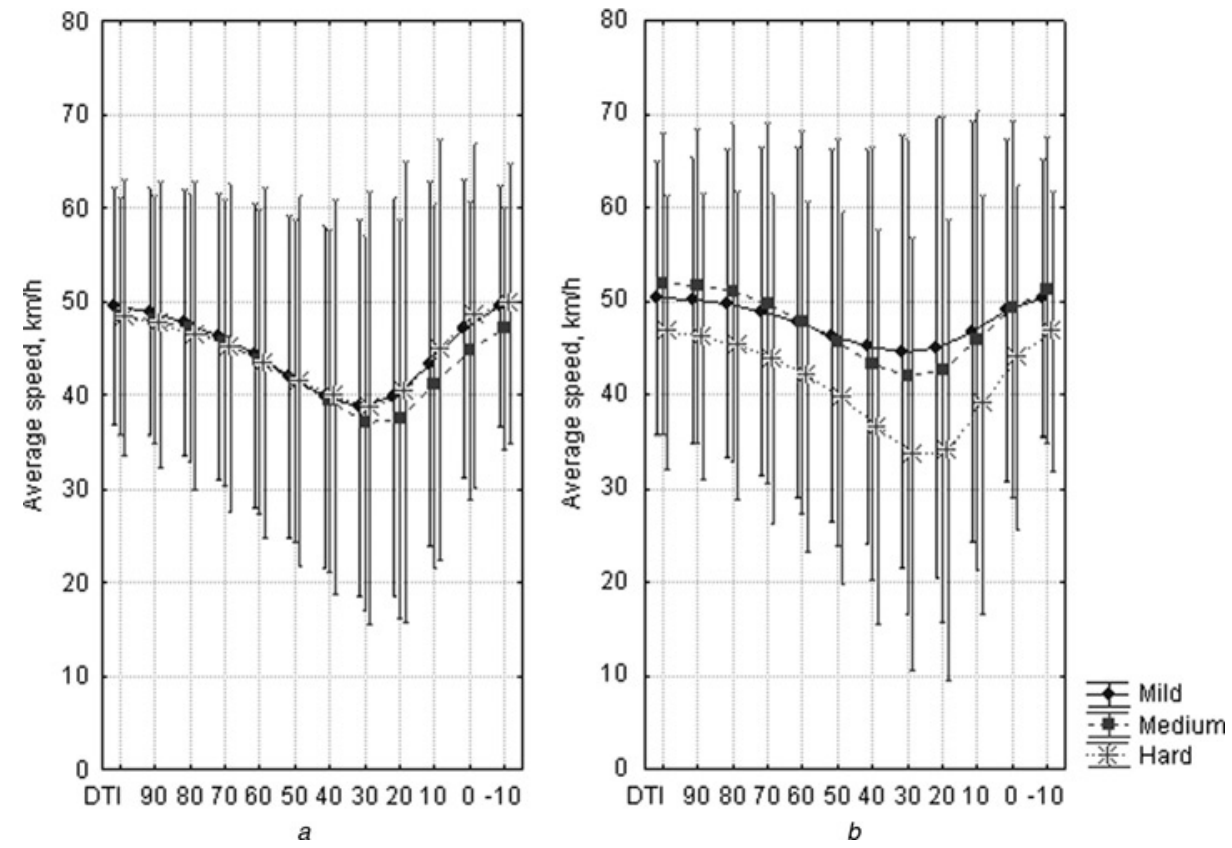

Figure 5 Average speed as a function of level of urgency and Distance to Intersection (DTI)

a Without additional task

$b$ With additional task 


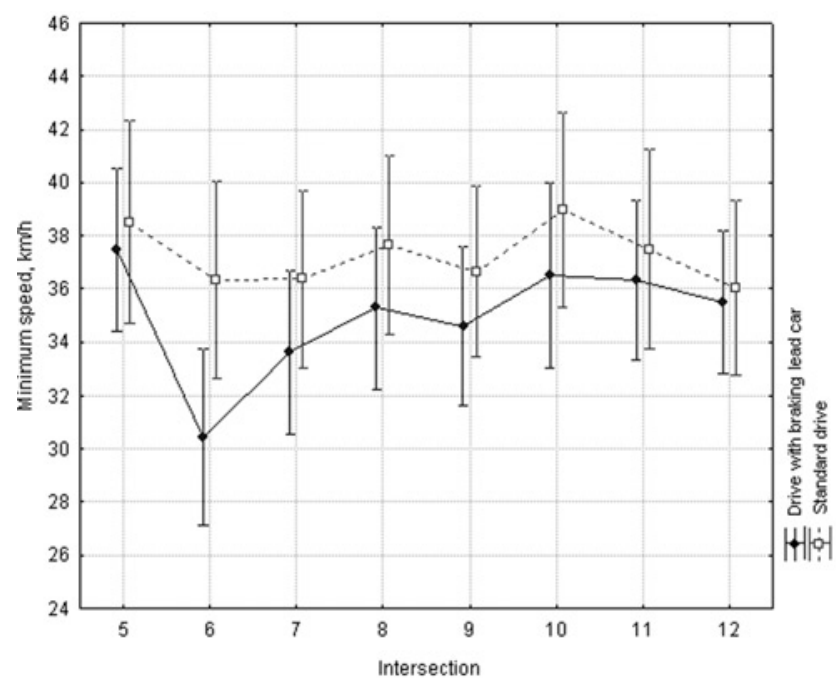

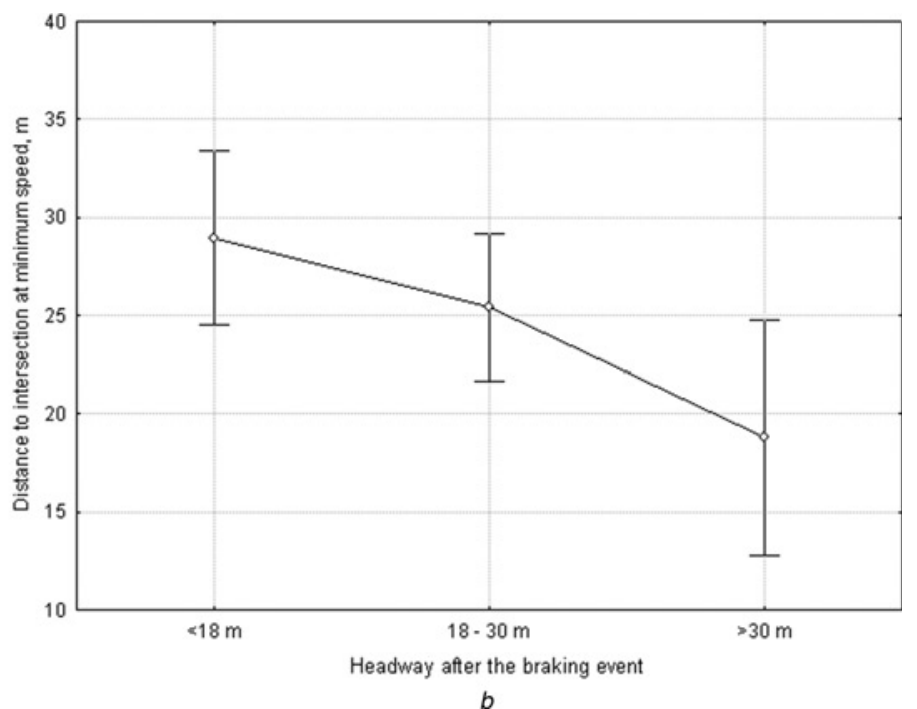

Figure 6 Minimum speed parameters

a Average minimum speed as a function of drive and intersection

$b$ Distance to intersection at minimum speed as a function of criticality level

Furthermore, the approach speed pattern changed significantly after the braking event, mostly for the participants with an additional task (Fig. 5). The participants without the additional task did not respond differently to varying levels of event urgency (Fig. 5a), whereas participants with an additional task showed a smoother approach speed pattern, except for those at the highest urgency level (hard), in which the acceleration curve was much more profound (Fig. 5b).

The urgency of the braking event had an additional effect on headway (a more serious event leading to a longer headway). Lateral position in the most urgent event was also different from that of the least urgent event $(F(1,13)=5.015$, $p<0.050)$, although this effect was not significant when all the three urgency levels were analysed $(F(2,22)=2.375$, $p=0.116)$. Only in the most urgent event did the drivers drive more to the right (i.e. safe) side of the road.

The correlation between the programmed urgency and the headway after the event (three levels of criticality) was $r=0.26 \quad(N=39)$, which was too small to deduce a positive relationship between the two variables. Therefore the analysis of parts of the data with this second variable was chosen. This second indicator caused a number of new effects to become statistically significant. The minimum speed decreased strongly after the braking event and then slowly increased to the normal value (Fig. 6a).

The distance from intersection at which the participants reached their minimum speed increased when the event was more critical $(F(2,33)=3.859, p<0.050$, Fig. $6 b)$. Furthermore, criticality had a statistically significant influence on the speed pattern and participants encountering the most critical events showed a more profound deceleration and acceleration profile than the participants who encountered the less critical braking event $(F(22,363)=4.318, p<0.001$, see Fig. 7).

There was a clear effect of workload on the time of the headway adaptation $(F(7,217)=2.238, p<0.050)$ in the event trial. Fig. 8 shows the different reactions between participants with and without an additional task. At the first intersection directly after the braking event, both participants with and without high workload showed a similar reaction to the braking lead car, but participants with an additional task normalised their headway directly after this intersection, whereas participants without this additional task drove with increased headway for a longer period of time.

Higher-criticality events often corresponded with higher driving speeds $(F(2,33)=5.769, p<0.010)$ and minimum

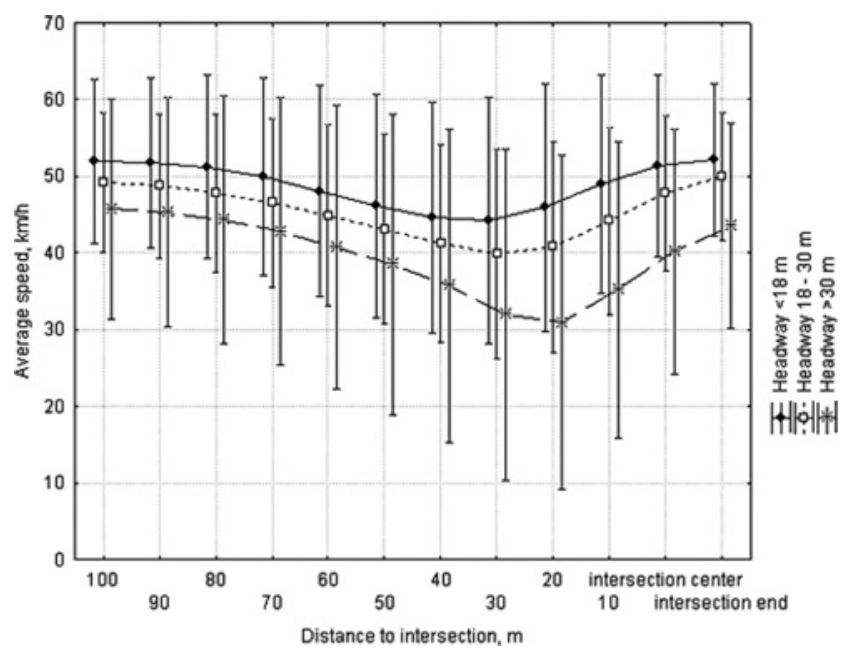

Figure 7 Average speed as a function of criticality level and distance to intersection 


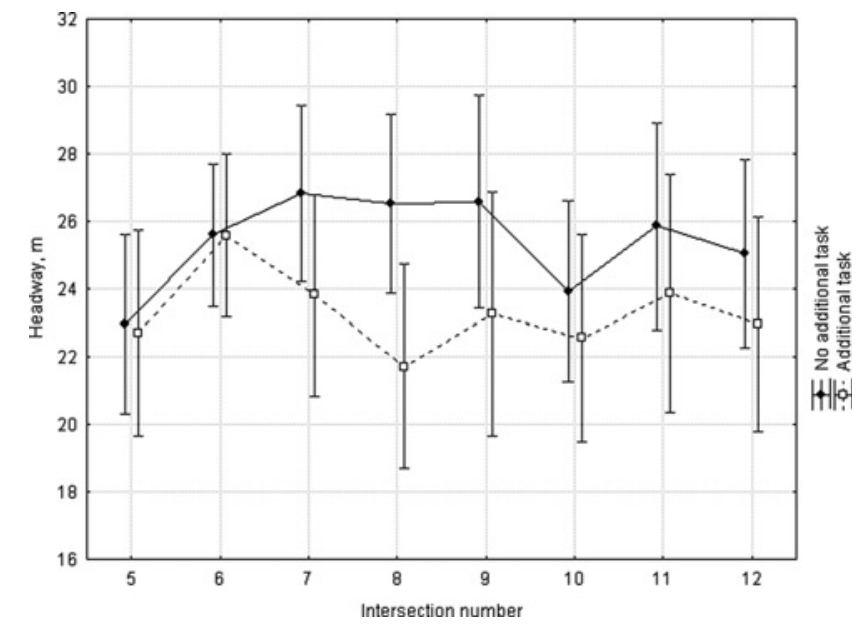

Figure 8 Headway as a function of workload and intersection

speeds $(F(2,33)=7.144, p<0.005)$, which could be related to the way the lead vehicle's behaviour was programmed. The lead vehicle would accelerate if the participant came too close, but only up to the participant's speed. Because of this speed/ headway relationship, participants driving at higher speeds would often have a less than average headway to the lead car and would therefore encounter more critical events. In further research, this criticality indicator should be established more clearly.

\section{Discussion and conclusions}

After an unexpected event, participants change their tactical driving behaviour. Average and minimum speeds are adjusted for the duration of a number of intersections after which their speed returns to normal. Participants show lower average and minimum speeds and later deceleration if the braking event happens further away.

Only the original urgency of the braking event (distance travelled by the participant during the braking time of the lead vehicle) significantly influenced the headway (i.e. was longer after more serious events) and lateral position (i.e. only drivers encountering the most serious events drove more to the right and safe side of the road). This was partially because of the fact that this indicator did not take into account how close to the lead car the participant was driving. Thus, another measure of criticality was determined, that is, the headway to the lead car after the event had occurred. This yardstick is more directly related to the possibility of a conflict. This new measure of criticality was demonstrated to be statistically significant for the minimum speed and the speed pattern. Higher speeds were also related to higher criticality. Lateral position was not influenced by this measure of criticality.

An additional cognitive task significantly increased driver workload. Overall speed patterns for drivers with an additional task were more smooth (smaller bandwidth of speed values) than for those without the additional task. The effects of the braking event on driving speed were most clear in the high-workload group, and a clear effect of interaction with event urgency was found in this group. On the one hand, the high-workload group with the highest urgency (hard) had lower speeds and a greater bandwidth of speed values than any of the other groups. On the other hand, the high-workload participants with the mild and medium urgency events showed higher overall speeds and enhanced smooth acceleration and deceleration than the group without an additional task. A possible explanation is that drivers with a high workload are more occupied with their additional task and therefore pay less attention to the unexpected event. They generally drive more smoothly, possibly because they respond less to certain cues in their environment and fall back on standard driving routines. However, when the unexpected event seems to reach a certain threshold, that is, its effect does get through, it leads to a strong change in behaviour. This is also seen in the effect on headway; participants with an additional task respond as strongly or more strongly to the braking event than participants without an additional task, but their behaviour adjustment is less protracted. Finally, drivers with a high workload tend to drive more to the left, that is, the less safe side of the road than those without. Hence, it can be concluded that not only urgency but also workload level has a significant effect on reactions to an unexpected event and that the effects of both factors interact.

The results help to understand driving behaviour under specific circumstances and may, as such, be an important step in the development of a workload manager as part of an ADAS that determines in which high-workload situations drivers need to be relieved of a part of their task. A promising result deduced is that drivers who are occupied by a secondary task respond differently to an unexpected event when compared with drivers who focus solely on their driving task. This is an important result in two ways, enhancing insight into the structure and relationship of the driving subtasks and helping to understand how workload affects the performance of drivers.

\section{Acknowledgments}

This research has been conducted within the research centre Applications of Integrated Driver Assistance (AIDA) of the Centre for Transport Studies at the University of Twente and TNO (The Netherlands), and as part of the Intelligent Vehicles project of TRANSUMO (TRANsition SUstainable Mobility), a Dutch platform for companies, governments and knowledge institutes that cooperate in the development of knowledge with regard to sustainable mobility. We gratefully acknowledge the support and contributions from Jeroen Hogema, Marika Hoedemaeker, Wytze Hoekstra, Antoon Wennemers, Marieke Martens and Ingmar Stel. The help of TNO Defence, Security and Safety (BU Human Factors, Soesterberg, The Netherlands) is acknowledged for allowing the authors to use its driving simulator to carry out their experiments. The authors thank three anonymous reviewers for their valuable comments. This paper is an extended version of a paper presented at 
the HUMANIST European Conference on Human Centred Design for Intelligent Transport Systems [23].

\section{References}

[1] Time series based on the previous accidents and network product 1976-2007, http://www.swov.nl/UK/ Research/Kennisbank/inhoud/kennisbank.htm, accessed May 2008

[2] BROOKHUIS K.A., DE VRIES G., DE WAARD D.: 'The effects of mobile telephoning on driving performance', Accid. Anal. Prev., 1991, 23, (4), pp. 309-316

[3] CHISHOLM S.L., CAIRD J.K., LOCKHART J.: 'The effects of practice with MP3 players on driving performance', Accid. Anal. Preven., 2008, 40, (2), pp. 704-713

[4] VAn driel C.J.G., hoedemaeker M., van ARem B.: 'Impacts of a congestion assistant on driving behaviour and acceptance using a driving simulator', Transp. Res. F, 2007, 10, (2), pp. $139-152$

[5] European Commission: Final report of the eSafety working group on road safety, Brussels, Belgium, 2002

[6] Ministry of Transport, Public Works and Water Management, Nota Mobiliteit; Naar een betrouwbare en voorspelbare bereikbaarheid, The Hague, The Netherlands, 2005

[7] DRAGUTINOVIC N., BROOKHUIS K.A., HAGENZIEKER M., MARCHAU V.: 'Behavioural effects of advanced cruise control use - a meta-analytic approach', European J. Transp. Infrastructure Res., 2005, 5, (4), pp. 267-280

[8] BROOKHUIS K.A., DE WAARD D., JANSSEN W.: 'Behavioural impacts of advanced driver assistance systems - an overview', European J. Transport and Infrastructure Res., 2001, 1, (3), pp. 245-253

[9] BROOKHUIS K.A., DE WAARD D.: 'ADAS acceptance and effects on behaviour: the consequences of automation?' in UNDERWOOD G. (ED.): 'Traffic and transport psychology: theory and application' (Elsevier, Amsterdam, 2005), pp. 273-278

[10] MICHON J.A.: 'Psychonomie onderweg'. Inaugural speech at University of Groningen, 2 November 1971

[11] RANNEY T.A.: 'Models of driving behavior: a review of their evolution', Accid. Anal. Prev., 26, (6), pp. 733-750

[12] aleXander G.J., LUnenfeld H.: 'Driver expectancy in highway design and traffic operations', Publication No
FHWA-TO-86-1, Federal Highway Administration, Washington, D.C., 1986

[13] RECARTE M.A., NUNES L.M.: 'Mental workload while driving: effects on visual search, discrimination, and decision making', J. Exper. Psychol. Appl., 2003, 9, (2), pp. 119-137

[14] FEENSTRA P., HOGEMA J.H.: 'Driving simulator assessment for human factors research'. Proc. 6th European Congress on ITS (ITS'07), Aalborg, Denmark, June 2007

[15] ST Software: 'Car driving simulator for driver training, assessment and research', http://www.stsoftware.nl, Accessed May 2008

[16] VAN Winsum W., Martens M., herLand L.: 'The effects of speech versus tactile driver support messages on workload, driver behaviour and user acceptance'. Report TM-01-D009, TNO Human Factors Research Institute, Soesterberg, The Netherlands, 1999

[17] HART S.G., STAVELAND L.E.: 'Development of NASA-TLX (task load index): results of empirical and theoretical research' in HANCOCK P.A., MESHKATI N. (EDS.): 'Human mental workload' (North Holland Press, Amsterdam, 1988), pp. 239-250

[18] VAN WinsUM W., HOEDemaeker M.: 'A road test of a prototype satellite system for in-vehicle menu control'. Report TM-00-C003, TNO Human Factors Research Institute, Soesterberg, The Netherlands, 2000

[19] DE WAARD D.: 'The measurement of drivers' mental workload'. Doctoral thesis, University of Groningen Groningen, The Netherlands, 1996

[20] Jahn G., oehme A., KRems J., gelau C.: 'Peripheral detection as a workload measure in driving: effects of traffic complexity and route guidance system use in a driving study', Transp. Res. F, 2005, 8, (3), pp. 255-275

[21] ANGELL L., AUfLICK J., AUSTRIA P.A., ET AL.: 'Driver workload metrics project task 2 final report', DOT HS 810 635, (National Highway Traffic Safety Administration, Washington, DC, 2006)

[22] OLSSON S., BURNS P.C.: 'Measuring driver visual distraction with a peripheral detection task', http://www-nrd.nhtsa. dot.gov/departments/nrd-13/driver-distraction/PDF/6.PDF, accessed May 2008

[23] SCHAAP T.W., VAN DER HORST A.R.A., VAN AREM B.: 'Influence of unexpected events on driving behaviour at different hierarchical levels: a driving simulator experiment'. Proc. European Conf. on Human Centred Design for Intelligent Transport Systems, Lyon, France, April 2008, pp. 131-140 